\title{
Atypical sarcoidosis diagnosed by bone marrow biopsy during renal workup for possible multiple myeloma
}

\author{
Amr El-Husseini • Alberto J. Sabucedo $\cdot$ Jorge Lamarche \\ Craig Courville $\cdot$ Alfredo Peguero
}

Received: 26 July 2012/ Accepted: 7 November 2012/Published online: 11 December 2012

(C) Japanese Society of Nephrology 2012

\begin{abstract}
Sarcoidosis is a multi-organ disease of unknown etiology characterized by non-caseating granulomas. Here we report the case of a 78-year-old white male with a past medical history of diabetes mellitus, hypertension, and chronic kidney disease stage III with a baseline serum creatinine of $2.5 \mathrm{mg} / \mathrm{dl}$. The patient had a prior admission history for acute kidney injury (AKI) attributed to dehydration and medication-induced nephro-toxicities. He presented to the renal clinic for follow-up with acute worsening of chronic kidney failure with a serum creatinine level of $3.5 \mathrm{mg} / \mathrm{dl}$. Examination revealed that he was anemic and mildly hypercalcemic with suppressed parathyroid hormone and had proteinuria of $1.3 \mathrm{~g}$ per day. The computed tomography scan of the abdomen revealed right renal pelvic non-obstructing calculi. Serum protein electrophoresis revealed gammopathy with two distinct monoclonal peaks consisting of immunoglobulin G (IgG) kappa and $\operatorname{IgG}$ lambda, respectively. The kappa/lambda ratio was within normal limits, and urine protein electrophoresis showed no evidence of a monoclonal peak or Bence Jones proteins. Further workup for multiple myeloma, including bone marrow (BM) biopsy, revealed polyclonal plasma cells and B cells with no clonality. No morphological and immunephenotypic evidence of plasma cell dyscrasia was found, but BM biopsy did show numerous non-caseating
\end{abstract}

A. El-Husseini · A. J. Sabucedo · J. Lamarche .

CraigCourville $\cdot$ A. Peguero

James A. Haley Veterans Hospital, University of South Florida,

Tampa, USA

\section{A. El-Husseini ( $\square)$}

Division of Nephrology, Bone and Mineral Metabolism,

University of Kentucky Chandler Medical Center, 800 Rose

Street, MN-564, Lexington, KY 40536-0298, USA

e-mail: amr.elhusseini.moh@uky.edu granulomas consistent with sarcoidosis. Skin biopsy from non-scaly 6-mm skin colored papule also showed non-caseating granulomas. The patient had elevated angiotensinconverting enzyme levels (165 ug/l) and an erythrocyte sedimentation rate of $27 \mathrm{~mm} / \mathrm{h}$. Kidney biopsy did not show granulomas. The hypercalcemia, proteinuria, and AKI responded well after 2 weeks of $60 \mathrm{mg}$ oral prednisone daily.

Keywords Sarcoidosis - Bone marrow - Proteinuria . Renal failure

\section{Introduction}

Bone marrow (BM) granulomas are identified in 17-53\% of patients with known sarcoidosis [1,2]. They may also be observed with disseminated fungal and mycobacterial infections. Although BM cultures were not performed in the case described herein, both acid fast bacilli and fungal stains were negative. Other causes of BM granulomas may include malignancy (particularly lymphoma), viral infection, drug or allergic reactions, and autoimmune diseases. Although BM biopsy has been used to diagnose sarcoidosis only in a very few cases reported in the medical literature $[3,4]$, our case demonstrates that this procedure may be a useful adjunct in establishing the diagnosis of sarcoidosis.

Sarcoidosis is a multi-organ-involved granulomatous disorder of unknown cause. Compared with the disease severity in the involved organs, nephropathy has a tendency to be mild [5]. However, Mayock et al. [6] reported that $14(9.7 \%)$ of 145 patients with sarcoidosis showed nephropathy, and, in Japan, Iwai et al. [7] reported that 14 $(27 \%)$ of 52 autopsy cases of sarcoidosis showed nephropathy. Therefore, sarcoidosis with nephropathy is 
not as uncommon as previously reported; however, glomerular involvement, especially immune complex glomerulonephritis, is unusual, and there has been no published description of whether and how this unusual type of glomerulonephritis and sarcoidosis may be related [8].

We report here a case of atypical sarcoidosis diagnosed by BM biopsy during the renal workup for a possible diagnosis of multiple myeloma with acute kidney injury (AKI).

\section{Case report}

Sarcoidosis is a multi-organ disease of unknown etiology characterized by non-caseating granulomas. Here we describe the case of a 78-year-old white male with a past medical history of diabetes mellitus, hypertension, and chronic kidney disease (CKD) stage III with a baseline serum creatinine concentration of $2.5 \mathrm{mg} / \mathrm{dl}$. The patient had a prior admission history for AKI attributed to dehydration and medication-induced nephro-toxicities. He presented to the renal clinic for follow-up with acute worsening of chronic kidney failure with a serum creatinine level of $3.5 \mathrm{mg} / \mathrm{dl}$. Examination revealed that he was anemic and mildly hypercalcemic with suppressed parathyroid hormone and had proteinuria of $1.3 \mathrm{~g}$ per day. A computed tomography scan of the abdomen revealed right renal pelvic nonobstructing calculi. Serum protein electrophoresis showed gammopathy with two distinct monoclonal peaks consisting of immunoglobulin $\mathrm{G}$ ( $\mathrm{IgG}$ ) kappa and $\mathrm{IgG}$ lambda, respectively (Fig. 1). The kappa/lambda ratio was within normal limits, and there was no evidence of a monoclonal peak or Bence Jones proteins on the urine protein electrophoresis. Further workup for multiple myeloma, including BM biopsy revealed polyclonal plasma cells and B-cells with no clonality. No morphological and immunophenotypic evidence of plasma cell dyscrasia was found, but BM biopsy did show numerous non-caseating granulomas consistent with sarcoidosis (Fig. 2). Skin biopsy from nonscaly 6-mm skin colored papule also showed non-caseating granulomas (Fig. 3). The Patient had elevated angiotensinconverting enzyme levels of $165 \mathrm{ug} / \mathrm{l}$ and an erythrocyte sedimentation rate of $27 \mathrm{~mm} / \mathrm{h}$. The kidney biopsy did not show granulomas, but did show focal, global glomerulosclerosis (40\% of glomeruli globally sclerotic) with moderate interstitial fibrosis, moderate hyperplastic arteriolosclerosis, and moderately severe arteriosclerosis. Mild acute tubulointerstitial nephritis was also detected. The renal biopsy was performed after the patient had initiated steriod treatment as a consequence of delays due to elevated International normalized ratio secondary to medications. Hypercalcemia, proteinuria, and serum creatinine improved after 2 weeks of therapy with $60 \mathrm{mg}$ oral

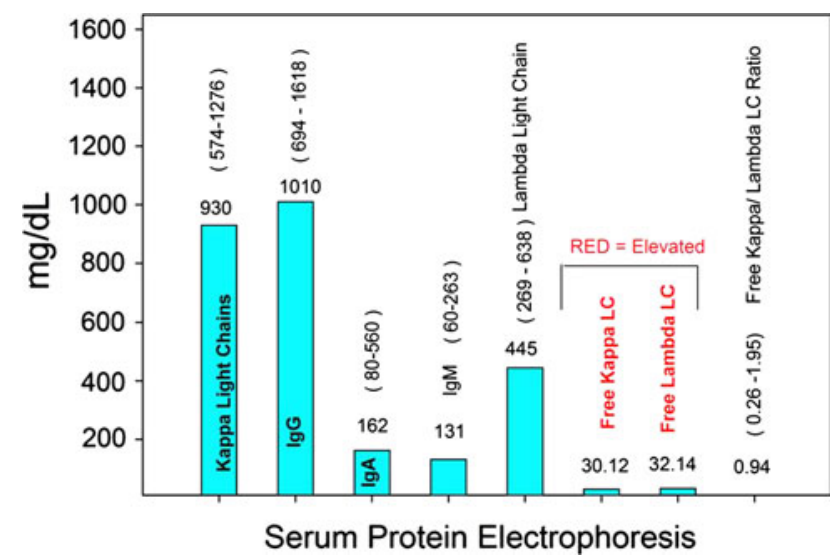

Fig. 1 Serum protein electrophoresis shows free kappa and lambda light chains $(L C)$ in gammopathy. The kappa/lambda chain ratio was within normal limits. $I g$ Immunoglobulin

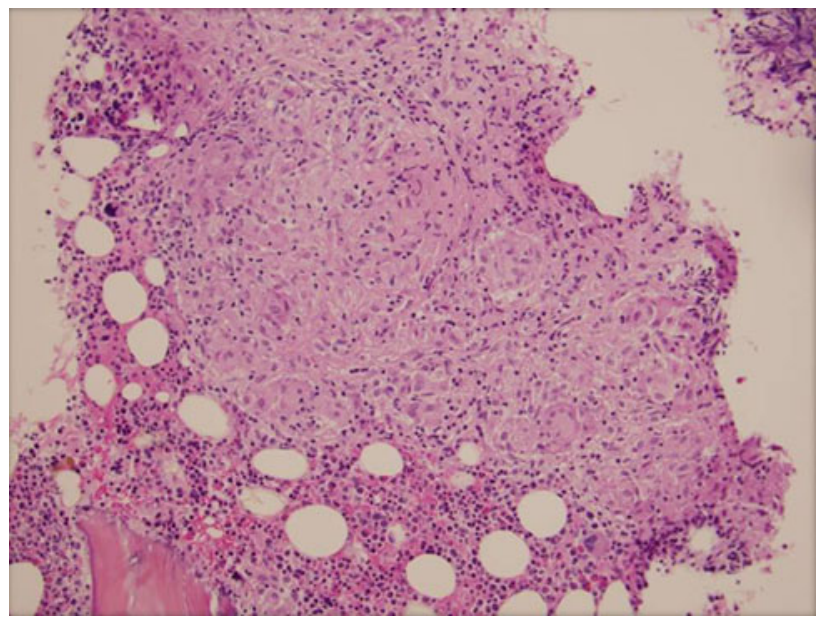

Fig. 2 Bone marrow biopsy showing evidence of granulomas

prednisone daily (Figs. 4, 5). The patient's glycemic control had been rather strict with multiple glycosylated hemoglobin measurements that were low (5\%). The patient's absolute blood glucose did bump during the treatment with steroids. The blood sugars went from a low of $100 \mathrm{mg} / \mathrm{dl}$ to almost $300 \mathrm{mg} / \mathrm{dl}$. The steroid therapy was only a short course of less than 1 month with the taper included. There were no red or white blood cell casts noted in multiple urine analyses performed during this admission. The urine analysis showed hyaline and granular cast, protein, and glucose. The patient's AKI was unlikely to have been the result of diabetes insipidus) secondary to neurosarcoidosis invasion of the hypothalamic centers given the absence of signs seen on the magnetic resonance imaging scan of the brain, normal serum sodium values (134-144 meq/l), and normal urine specific gravity, all of which do not support diabetes insipidus as an etiology of the AKI. The patient's chest $\mathrm{X}$-ray was notable for signs of bilateral lymphadenopathy in 


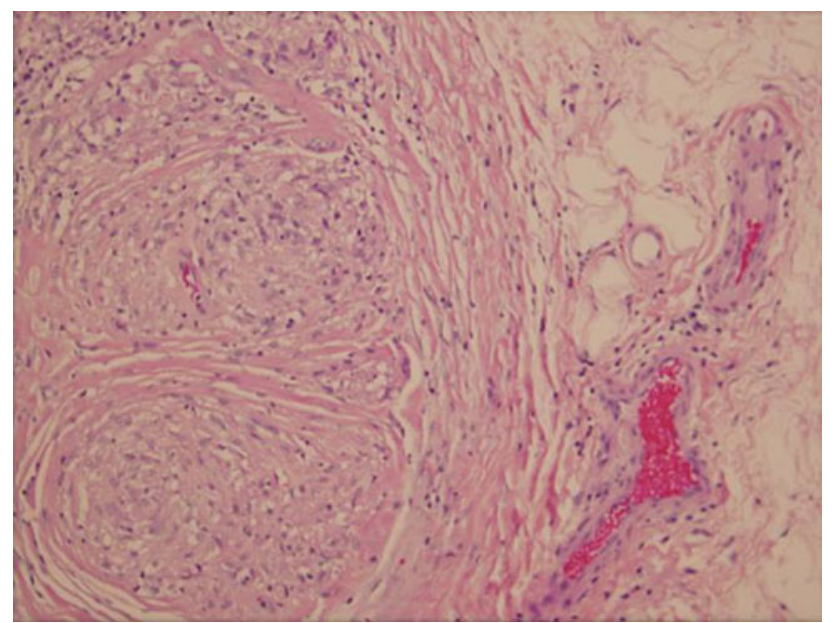

Fig. 3 Skin biopsy showing non-caseating granulomas

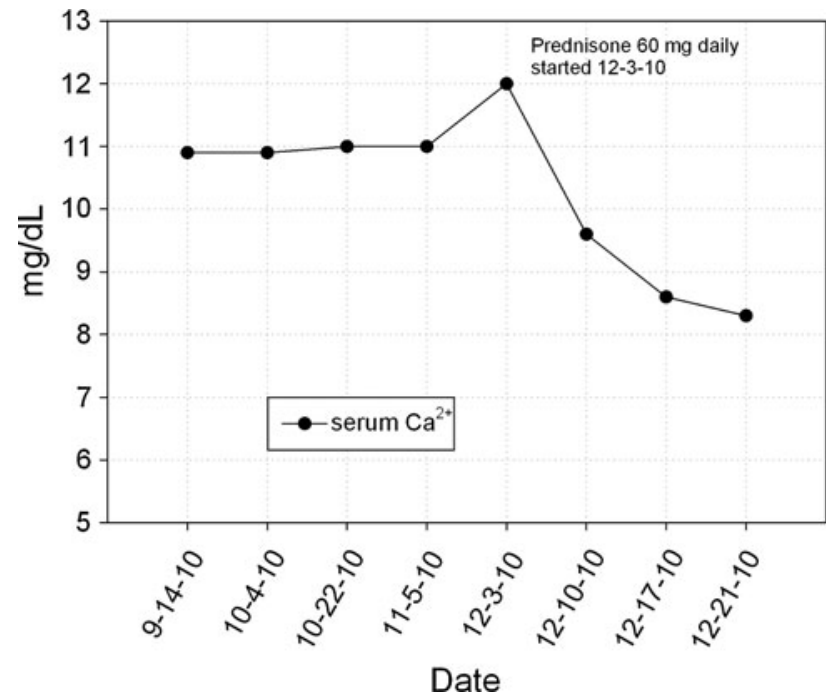

Fig. 4 Corrected calcium levels during the disease course

the hilar regions, and a CT scan of the chest showed increases in subcarinal and bilateral hilar lymph nodes.

\section{Discussion}

Sarcoidosis is a chronic multi-system disease of unknown cause, characterized by the accumulation of non-caseating granulomas in any organ, but most commonly in the lung. The kidney is mainly involved as calcemic nephropathy due to metabolic calcium abnormalities (including hypercalcemia, hypercalciuria, nephrocalcinosis, and nephrolithiasis), tubulointerstitial nephropathy with granulomatous infiltration and, rarely, glomerulopathy [9].

Sarcoidosis is associated with an elevated overall relative risk for the development of malignancies, including

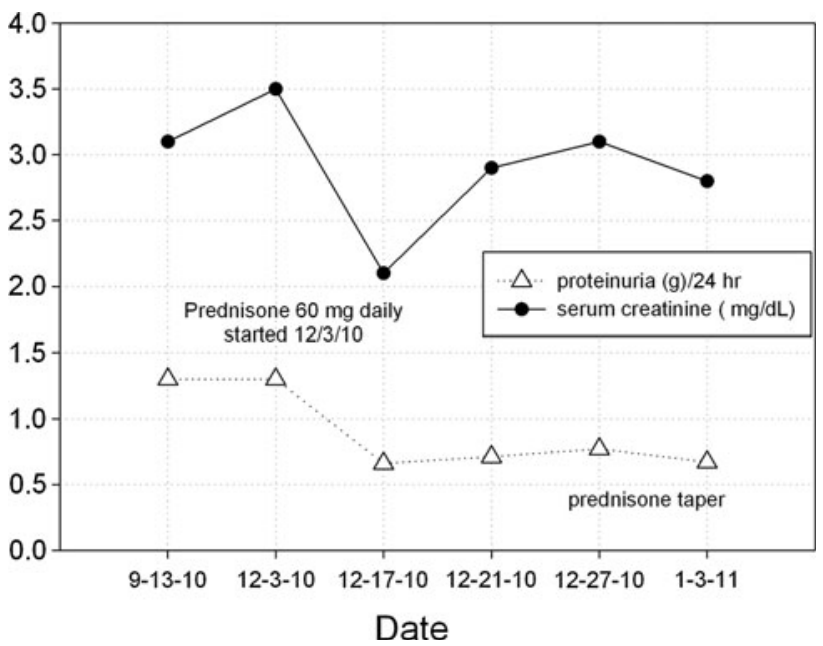

Fig. 5 Serum creatinine and proteinuria levels during the disease course

lymphomas and solid tumors [10-13]. The association of multiple myeloma with sarcoidosis is rare and has been reported in only 14 patients, 13 of whom had BM involvement with multiple myeloma and one had an extramedullary plasmacytoma of the mesentery [14]. Two of the patients were diagnosed with simultaneous sarcoidosis and multiple myeloma $[15,16]$. Sarcoidosis represents a chronic disease of immunologic disturbance. This disturbance has been proposed to explain the relationship between sarcoidosis and lymphoid malignancies, and it is possible that a similar mechanism may lead to the development of other hematologic malignancies, including multiple myeloma [14]. Polyclonal hyper-gammaglobulinemia is a common feature in sarcoidosis patients [17] and may be due to continuous stimulation of $\mathrm{B}$ cells by activated CD4+ $\mathrm{T}$ cells. In addition, this chronic stimulation may result in prolonged B-cell and plasma cell halflives that may increase the risk of genetic mutations, leading to the formation of a malignancy, such as multiple myeloma [18].

In 2002, Sen et al. reported that 11 cases of sarcoidosis and multiple myeloma had been published (including their own case report) and that three of the 11 patients had monoclonal gammopathy of undetermined significance preceding the onset of multiple myeloma. In most of the reported cases, sarcoidosis preceded the development of multiple myeloma [19]. Sarcoidosis is associated with disturbances of the immune system, including decreased CD8-positive $\mathrm{T}$ cell suppressor/cytotoxic cells, activation of CD4-positive $\mathrm{T}$ cell helper/inducer cells, abnormal cytokine production, cutaneous anergy to particular antigens, such as tuberculin purified protein derivative, and hypergammaglobulinemia [20]. 
BM granulomas have been identified in 17-53\% of patients with known sarcoidosis [21]. BM granulomas may also be observed with disseminated fungal and mycobacterial infections. Although BM cultures were not performed in our case, both acid fast bacilli and fungal staining was negative. Other causes of BM granulomas may include malignancy (particularly lymphoma), viral infection, drug or allergic reactions, and autoimmune diseases. Although $\mathrm{BM}$ biopsy has been used to diagnose sarcoidosis in only a very few of the published cases [22], this case demonstrates that this procedure may be a useful adjunct in establishing the diagnosis of sarcoidosis.

Kidney involvement occurs in a variety of ways. The epithelioid cells of the granulomas may produce calcitriol, with the result that sarcoid patients may develop hypercalciuria, nephrocalcinosis, nephrolithiasis, and hypercalcemia. Renal interstitial injury may occur with or without granuloma formation. Gobel and his colleagues [9] postulated that there are reasons to believe that the chronic hypercalcemia and hypercalciuria that accompany sarcoidosis can lead to renal insufficiency.

The patient's hypercalcemia, proteinuria, elevated ACE levels, and positive bone marrow biopsy point to sarcoid disease, which is a rare cause of acute renal injury. Although the patient's kidney biopsy findings were not suggestive of sarcoidosis, the absence of sarcoid granulomas in the kidney biopsy may be explained by the use of relatively high doses of prednisone $(60 \mathrm{mg}$ daily for 2 weeks) before the biopsy. Preemptive corticosteroid use may also help explain the fact that the presence of acute tubulointerstitial nephritis was mild in our patient. Nevertheless, the finding of non-caseating granulomas in the renal interstitium is a rare finding. In addition, the presence of interstitial nephritis is not specific. Overall, renal involvement is uncommon when the activity and symptoms of the disease in other organ involvement is relatively quiescent, as in our patient.

Therefore, a high index of suspicion to diagnose sarcoidosis must be present in cases where AKI is present with hypercalcemia. The medical history of recurrent AKI in our patient was attributed to be secondary to dehydration and medication-induced renal toxicities. The patient always presented with very mild elevations in serum calcium, which led us to theorize that these elevations were the result of dehydration, and not vice versa. As the patient's chemistries and renal function improved with intravenous hydration administration, the possibility of sarcoid-induced interstitial nephritis was not given much attention. Additionally, even if a kidney biopsy were to be performed during an AKI episode, any possibility of the results leading to a diagnosis was quite likely to be low without a proper workup for the hypercalcemia and proteinuria. The diagnosis of sarcoidosis was not possible without doing a BM biopsy. Thus, as stated above, a high suspicion of sarcoidosis is imperative in cases of AKI with hypercalcemia due to its multiple atypical presentations. A complete workup for proteinuria may also help to detect unrecognized masked rare diagnoses.

Although BM biopsy has been used to diagnose sarcoidosis in only a very few cases presented in the medical literature, our case demonstrates that this procedure may be a useful adjunct in establishing the diagnosis. Elevated angiotensin-converting enzyme levels may serve as a good screening test, but the demonstration of non-caseating granulomas on BM confirms the final diagnosis in the proper clinical context. Every effort should be made to diagnose this condition because it is treatable with steroids or cytotoxic agents, such as methotrexate. In our patient, hypercalcemia and AKI reversed with steroid treatment.

\section{Conflict of Interest None.}

\section{References}

1. Bodem CR, Hamory BH, Taylor HM, et al. Granulomatous bone marrow disease: a review of the literature and clinicopathologic analysis of 58 cases. Medicine. 1983;62:372-82.

2. Browne PM, Sharma OP, Salkin D. Bone marrow sarcoidosis. JAMA. 1978;240:2654-5.

3. Levy TM, Blundell E, Slade R, et al. Diagnosis of sarcoidosis by bone marrow trephine biopsy. Br J Haematol. 1993;84:179-91.

4. Rayner CK, Burnet SP, McNeil JD. Osseous sarcoidosis: a magnetic resonance imaging diagnosis. Clin Exp Rheumatol. 2002;20:546-8.

5. Kanamori H. IgM-immune complex glomerulonephritis associated with Sarcoidosis. Clin Exp Nephrol. 2006;10:68-73.

6. Mayock RL, Bertrand P, Morrison CE, Scott JH. Manifestation of sarcoidosis. Analysis of 145 patients, with a review of nine series selected from the literature. Am J Med. 1963;35:67-89.

7. Iwai K, Tachibana T, Matsui Y, Shigematsu S, Izumi T. Statistical and pathological observations of autopsy cases of sarcoidosis. Nihon Kyoubu Sikkan Gakkai Zassi. 1973;11:749-63.

8. Kanamori H, Ota M, Takeoka H, Osafune K, Yonezu S, Fukatsu A, Kanatsu K. IgM-immune complex glomerulonephritis associated with sarcoidosis. Clin Exp Nephrol. 2006;10(1):68-73.

9. Gobel U, Kettritz R, Schneider W, Luft FC. The protean face of renal sarcoidosis. J Am Soc Nephrol. 2001;12:616-23.

10. Brincker $\mathrm{H}$, Wilbek $\mathrm{E}$. The incidence of malignant tumours in patients with respiratory sarcoidosis. Br J Cancer. 1974;29:247-51.

11. Brincker H. Sarcoidosis and malignancy. Chest. 1995;108:1472-4.

12. Cohen PR, Kurzrock R. Sarcoidosis and malignancy. Clin Dermatol. 2007;25:326-33.

13. Landgren O, Bjorkholm M, Montgomery SM, et al. Familial characteristics of autoimmune and hematologic disorders in 8,406 multiple myeloma patients: a population-based case-control study. Int J Cancer. 2006;118:3095-8.

14. Brown G, Shapeero LG, Weiss BM, Roschewski M. Multiple myeloma with lacrimal gland amyloidosis and sarcoidosis. Am J Hematol. 2010;85(7):506-9.

15. Selroos O, Brander L, Virolainen M. Sarcoidosis and myeloma of lambda-type IgG. Acta Med Scand. 1974;195(1-2):59-63.

16. Pettersson T, Koivunen E, Ilvonen M, et al. Sarcoidosis and multiple myeloma: an association. Br Med J (Clin Res Ed). 1987;295:958. 
17. Leibovitch I, Selva D, Goldberg RA, et al. Periocular and orbital amyloidosis: clinical characteristics, management, and outcome. Ophthalmology. 2006;113:1657-64.

18. Brincker H. The sarcoidosis-lymphoma syndrome. Br J Cancer. 1986;54:467-73.

19. Sen F, Mann KP, Medeiros LJ. Multiple myeloma in association with sarcoidosis. Arch Pathol Lab Med. 2002;126:365-8.

20. Vourlekis JS, Sawyer RT, Newman LS. Sarcoidosis: developments in etiology, immunology and therapeutics. Adv Intern Med. 2000;45:209-57.
21. Garwood A, Mikuls T. A case of isolated sacral and pelvic sarcoidosis diagnosed by bone marrow biopsy. J Clin Rheumatol. 2003;9:321-4.

22. Rayner CK, Burnet SP, McNeil JD. Osseous sarcoidosis: a magnetic resonance imaging diagnosis. Clin Exp Rheumatol. 2002;20:546-8. 